\title{
Complication rates for central venous catheters used for parenteral nutrition
}

\author{
P. Dave, A. F. Cartwright and J. M. Subhani \\ Basildon and Thurrock University Hospital NHS Foundation Trust, Basildon, Essex SS16 5NL, UK
}

Central venous catheters (CVC) are often used in unstable patients for monitoring, resuscitation and delivery of drugs in a life-threatening situation. In these scenarios, the small risks of the CVC are clearly outweighed by the immediate advantages. The delivery of parenteral nutrition (PN) via a CVC is less immediately life-saving and so the risks must be more clearly assessed and minimised. A local revision of elective CVC placement protocol led to a change in practice in 2006. This limited who and where CVCs were placed and managed. This change in protocol was subsequently ratified in the epic2 guidelines ${ }^{(1)}$.

This study examined the complications of CVC use for PN with particular regard to infection rates and the success and outcome of feeding.

This was prospective audit of all CVCs inserted from the 2004 to 2007 that were used for PN. Catheter related blood stream infection (CRBSI) was determined by the clinical team. This was usually if either (i) positive "tip" and blood cultures for the same organism or (ii) positive culture (tip or blood) with a clinical response to removal.

Two hundred and thirty seven patients ranging from 16 to 93 (avg. 64.5 years old) had 337 CVCs inserted. The majority were triplelumen (242) and 13 were tunnelled. Seventy five percent of patients' primary diagnosis was either an upper or lower gastrointestinal pathology. Sixty-four percent of CVCs were placed into the right internal jugular.

Complications were uncommon: failed at insertion $1.2 \%$, misplaced CVC tip 3\%, pneumothorax $0.3 \%$ and no arterial punctures. There were 55 suspected CRBSIs, but $20 \%$ had no tip culture sent. Eleven out of 55 were proven CRBSI. In those not suspected of CRBSI, a tip culture was sent in $85(30 \%)$. In 11/85 (13\%) of these an "unsuspected" CRBSI was proven.

The suspicion of a CVC infection did not statistically effect the likelihood of a CRBSI $(P=0.23)$.

The rate of CRBSI/1000 CVC days varied with time from insertion (Table 1).

\begin{tabular}{lc}
\multicolumn{2}{c}{ Table 1. CRBSI rates with time interval from placement } \\
\hline Days from insertion & Rate of CRBSI/1000 CVC days \\
\hline $0-4$ & 1.6 \\
$5-9$ & 13.5 \\
$10-20$ & 2.1 \\
$20-40$ & 3.2 \\
\hline
\end{tabular}

Table 2. Who placed the CVC and CRBSI in the different years of the study

\begin{tabular}{lccc}
\hline Year & $2004 / 5$ & 2006 & 2007 \\
\hline Total CVCs & 82 & 144 & 108 \\
Placed by & & & $100(93 \%)$ \\
-Anaesthetist & $48(59 \%)$ & $131(91 \%)$ & $3(3 \%)$ \\
-Radiologist & $5(6 \%)$ & $5(4 \%)$ & $7(6.5 \%)$ \\
CRBSI & $7(8.5 \%)$ & $8(5.5 \%)$ & 5.5 \\
CRBSI/1000 CVC days & 6.6 & 5.2 & \\
\hline
\end{tabular}

The average length of use per CVC was 11.6 days. Overall $69 \%$ of CVC were used until the patient completed PN and either restarted oral feeding or an alternative feeding route and $18 \%$ of patients died. The majority of the rest of the CVC were either removed for suspected CRBSI or accidentally. Only $25 \%$ of CVCs were used for less than 5 days of PN. The commonest reasons for termination of feeding in this group were "end of treatment" (44\%), alternative feeding route (20\%) and died (19\%).

Complications of CVC insertion were rare. CRBSI occurred in $6.5 \%$ and half the time was not suspected. The peak incidence of CRBSI was 5-9 days after insertion Table 1. This peak may imply other factors than routine handling of CVC's are causative. This may include the nature of the insertion or the clinical state of the patient in the first 10 days (i.e. bacteraemia). There has been a marked shift in who and where the CVCs have been inserted but without any obvious effect on CRBSI rates Table 2. In view of this lack of response, additional measures such as chlorhexidine impregnated CVC's and a chlorhexidine impregnated patch dressing are being introduced to the Trust.

1. epic2: National Evidence-Based Guidelines for Preventing Healthcare Associated Infections in NHS Hospitals in England (2007) $J$ Hosp Infect 655, S1-S64. 